\section{Enfoque de género: De la teoría a la práctica en la implementación de politicas públicas para la paz en Cartagena}

Gender approach: From theory to practice in the implementation of public policies for peace in Cartagena

Bexi Katerine Cruz-Torrado

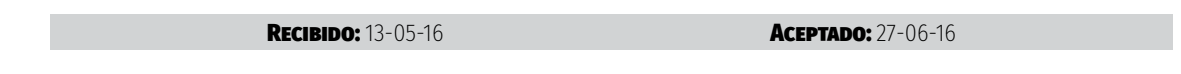

Resume

El presente artículo de investigación en curso responde al proyecto "Enfoque de género en la implementación de politicas públicas para la paz en la ciudad de Cartagena" que pretende establecer estrategias para la incorporación del enfoque de género en la implementación de políticas públicas para la paz en el marco del acuerdo final para la terminación del conflicto y la construcción de una paz estable y duradera entre el
Gobierno colombiano y las FARC-EP, en la ciudad de Cartagena. La metodología utilizada en la ejecución del proyecto es de enfoque cualíativio con un diseño de investigación fenomenológicacautitizando la revisión
documental (parte central del presente artículo), la observación yentrevista como técnicas de investigación Palabras clave: enfoque de género, politicas públicas y paz.

Abstract

This article responds to the research project "Gender focus in the implementation of public policies for peace in the cily of Cantagena which aims to establsh stralegles for incorporating the gender approach ermination of the confict and the construction of a stable and durable parce between the Colombia government and the FARC-EP, in the city of Cartagena. The methodology used in the execution of the project is a qualitative approach with a phenomenological research design, using documentary review (central part

Keywords: gender approach, public politics and peace.

Trabajadorara Social, candidata a Magister de la Maestria en Conflicto Social y Construcción de Paz de la Universidad de Cartagena Comfenaliton beruz@itecnologicocoménenalo.e.edu.co
El enfoque de género en las negociaciones entre el Gobierno Colombiano y las Fuerzas Armadas Revolucionarias de Colombia -Ejército del Puebloen adelante FARC-EP, fue un tema neurálgico que se convirtió en una de las principales razones por la cual se realizó oposición al proceso, pero además evidenció la resistencia que ocasiona solo mencionarlo. Pues, al hablar de género y todo lo que ello implica se transgrede el orden históricamente constituido de distribución sexual de los roles, de ejercicio y control del poder, así como de la sexualidad.

De allí que se requiere dar un abordaje desde la teoría a la compresión social del género y cómo en la práctica se ha hecho uso del enfoque, partiendo de la premisa de que la paz solo puede ser concebida en la medida que se responde a la garantía de los derechos para todas las poblaciones, por tanto que no se pueda iniciar procesos de construcción de paz sobre la base de la exclusión o la discriminación de los grupos sociales ya sea basados en estereotipos de sexo, género, raza, orientación sexual, edad, clase social o cualquier otra.

En esa medida en el presente documento, se establecen estrategias para la incorporación del enfoque de género en la implementación de políticas públicas - en adelante PP para la paz en el marco del acuerdo final para la terminación del conflicto y la construcción de una paz estable y duradera en la ciudad de Cartagena, a partir de la revisión de los elementos constitutivos de género que se plantean en las PP; en una segunda etapa del proceso investigativo se espera responder a la identificación del abordaje de enfoque de género que están utilizando las entidades públicas y organizaciones sociales de mujeres y de la población LGBTI2 que acompañan procesos de paz en la ciudad y asi determinar nuevas estrategias para la incorporación del enfoque de género.

\section{DESCRIPCIÓN DEL PROBLEMA DE INVESTIGACIÓN}

Históricamente el concepto de género ha sido asociado erróneamente a las mujeres, dado que es un tema abordado inicialmente desde los movimientos feministas que plantean la necesidad de hacer un análisis de las relaciones exaltando la diversidad de la población, esto incluye el sexo, género, etnia

\footnotetext{
2 Lesbianas, gays, bisexules, trans (transgeneristas, transexuales, travestis o transformistas) e intergeneristas.
} 
discapacidad u otras. Partiendo de esa asociación género-mujeres, se ha creído que entonces los análisis para su implementación abarcan solo a las mujeres y en algunos casos a la población LGBTI.

Pareciera entonces que el enfoque de género se cumple solo con agregar la palabra mujer a los diversos procesos, proyectos o políticas o con desagregar algunos datos de las acciones realizadas por la categoría sexo. Esto contribuye a sesgar y limitar el enfoque a no visionar problemáticas relacionadas con el patriarcado que afecten en general a la población, aunque en forma diferente.

En la práctica, materializar el enfoque no es tan fácil, se puede tender a generalizar en la transversalización, por ejemplo, al asociar género a familia o mujeres o incluso a re-victimizar cuando se ignoran las causas históricas, culturales, políticas que soportan la discriminación y no se tienen en cuenta las particularidades de las problemáticas que aquejen a la población.

Por otra parte, el desconocimiento del género como categoría de análisis, de lo que comprende y de lo que realmente es, generó en Colombia, por ejemplo, diferentes manifestaciones sociales en contra de la mención del mismo en el acuerdo final entre el Gobierno colombiano y las FARC-EP, lo que evidenció el inconformismo de gran parte de la población frente a lo que implica su utilización, pero además confirmó que no existe claridad conceptual acerca del término.

En Colombia este enfoque se ha estado implementado desde antes de las negociaciones entre el Gobierno colombiano y las FARC-EP, dado que esto ha sido una directriz que Colombia ha asumido con la ratificación de los diferentes instrumentos internacionales que garantizan los derechos de las mujeres, asi mismo a nivel nacional con la adopción de algunas leyes como la ey 1257 de 2008 sobre no violencias contra las mujeres, la 1719 de 2014 sobre victimas de violencia sexual y la 1761 de 2015 sobre feminicidios y algunas sentencias de la Corte Constitucional o el Consejo de Estado, aluden sobre la apremiante necesidad de poner en práctica el enfoque de género.

Todo lo anterior lleva a preguntarse, ¿cómo incorporar el enfoque de género en la implementación de políticas públicas para la paz en el marco del acuerdo final para la terminación del conflicto y la construcción de una paz estable y duradera en la ciudad de Cartagena?

\section{METODOLOGIA}

El proyecto de investigación en curso aborda un enfoque de investigación cualitativa en la medida que la realidad sujeto de investigación parte de la subjetividad de quienes le abordan o han trabajado en el tema para llegar a nuevos significados y plantear categorías emergentes que permitan realizar un análisis holístico del tema de estudio tomando como base las particularidades desde las cuales se ha manejado el enfoque de género. Por su parte, el diseño de investigación utilizado es el fenomenológico en tanto analiza los significados, comportamientos y narrativas inmersos en el discurso sobre enfoque de género utilizado por las entidades públicas y organizaciones sociales de mujeres y de la población LGBTI que acompañan procesos de paz en la ciudad.

Las técnicas de investigación utilizadas son la revisión documental relacionada con documentos de política pública sobre paz y enfoque de género, la observación y la entrevista para llegar a categorías emergentes e identificar el abordaje y las estrategias utilizadas en el marco de la comprensión e implementación del enfoque de género. La población sujeto de estudio son entidades públicas, organizaciones sociales de mujeres y de la población LGBTI que defienden los derechos humanos, acompañan o tienen trabajo concerniente a la paz

\section{DISCUSIÓN Y RESULTADOS}

\section{Enfoque de género}

Hablar de enfoque de género supone comprender primero los conceptos de sexo, género, patriarcado, equidad de género, perspectiva y teorías de género, dado que el enfoque es una herramienta de política pública que se nutre de las reflexiones que se han venido dando a lo largo de la historia en relación a las formas de comportamiento socialmente enseñadas y aceptadas.

El género es definido como una construcción que la sociedad ha realizado sobre las formas de comportarse, actuar, ver e imaginar el mundo según se es hombre o mujer, esta identificación de los seres humanos ha traído consigo formas históricas de relación y distribución del poder desiguales, basadas en la supuesta superioridad de hombres sobre las mujeres, lo que se constituye un sistema patriarcal basado en prácticas sexistas de convivencia. 
Para Facio (s.f.) el género “... alude, tanto al conjunto de características y comportamientos, como a los roles, funciones y valoraciones impuestas dicotómicamente a cada sexo a través de procesos de socialización, mantenidos y reforzados por la ideología e instituciones patriarcales..." (p.13). En ese sentido vale la pena aclarar que el sexo hace referencia exclusivamente a las diferencias biológicas de los seres humanos y se convierte en un elemento constitutivo sobre el cual el sistema patriarcal pone en situación de desventaja a la mujer sobre el hombre.

Partiendo de estas consideraciones, el género es entonces una categoría que permite hacer un análisis a las relaciones y cómo históricamente a través de la cultura, la educación, las normas, la religión, la filosofía y la estructura de la sociedad se ha discriminado y subordinado a la mujer frente al hombre, basado en las diferencias biológicas.

Esto, ha permitido que se justifiquen, toleren, naturalicen, reproduzcan y continúen situaciones como las discriminaciones y violencias contra las mujeres, las brechas salariales y los estereotipos de género que redundan en los diferentes ámbitos de la vida familiar, social, espiritual, política, económica e incluso en la salud de las mujeres.

Sin embargo, es preciso señalar que el género también involucra el ejercicio de la sexualidad y el reconocimiento de cómo cada persona se ve en el mundo, se identifica y define, de alli que incluye las orientaciones sexuales $y$ las identidades de género ${ }^{3}$.

Otro concepto que soporta lo ya explicado, es el de patriarcado, que hace referencia a un sistema social, político y estructural que discrimina, oprime y genera violencia sobre las mujeres y personas que no siguen una conducta hetero-normativa, es decir, sobre quienes conciben su sexualidad e identidad distinta a la heterosexual.

Es importante señalar, que el patriarcado afecta a hombres y mujeres de manera distinta, pero afecta a todos, usualmente es más fácil identificar cuando se afecta al género femenino o a la población LGBTI, pero difícil-

3 Para Caribe Afirmativo (2010), Identidades de género corresponde a "las construcciones culturales en torno a la sexulalidad...

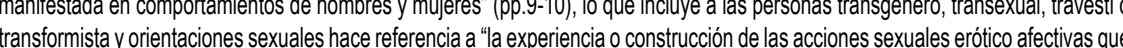
tanto hombres como mujereses desarorolan en el ámbito sexual hacia los demás" (p.10) dentro de ellas se encuentra ser heterossexval, gay, lesbiana o bisexual. mente se reconoce cuando se está afectando a los hombres heterosexuales. A manera de ejemplo se puede mencionar que el patriarcado afecta a los hombres al generalizar y creer que todos son violentos, agresivos o incapaces de cuidar de los hijos e hijas. Al respecto Facio (s.f.) plantea:

Las ideologias patriarcales no solo afectan a las mujeres al ubicarlas en un plano de inferioridad en la mayoría de los ámbitos de la vida sino que restringen y limitan también a los hombres, a pesar de su estatus de privilegio. En efecto, al asignar a las mujeres un conjunto de características, comportamientos y roles "propios de su sexo", los hombres quedan obligados a prescindir de estos roles, comportamientos y características y a tensar al máximo sus diferencias con ellas. (p.3)

Como respuesta para abordar este sistema, las mujeres a través de los movimientos sociales, redes, organizaciones o de manera personal, empiezan a cuestionar las formas de relacionamiento que históricamente se han presentado, ponen en la palestra pública conceptos como género, patriarcado, equidad, perspectiva y enfoque de género, de alli que fácilmente se haga la relación género igual a mujer.

Ahora bien, la equidad de género hace referencia al equilibrio entre la justicia y la igualdad para hombres y mujeres, reasignándole a la mujer lo que le corresponde en cuestión de derechos. Por su parte, la perspectiva de género hace alusión al análisis y la mirada crítica de las relaciones de poder y subordinación entre hombres y mujeres que han sustentado la forma en que se ha construido la sociedad, tiene mucha relación con desentrañar como se han dado las relaciones entre los sexos. En palabras de Facio (s.f.), la perspectiva de género permite:

...visibilizar la realidad que viven las mujeres así como los procesos culturales de socialización que internalizan y refuerzan los mecanismos de subordinación de las mujeres... (p.10), ...Ponen a descubierto cómo el hombre y lo masculino son el referente de la mayoría de las explicaciones de la realidad. ...introduce la mirada y experiencia del género femenino... (p.16)

Todo lo anterior pone en consideración que las teorías de género no son un concepto nuevo, ni poco teorizado, por el contrario, evidencian los estudios, reflexiones, investigaciones, que se han realizado a lo largo de los años por la 
incorporación del enfoque de género en la actuación política, social, cultural, educativa y estructural

En lo relativo al enfoque de género, es un principio porque orienta una actuación en materia de política pública, criterio porque permite un análisis y es una herramienta en la medida que brinda unas directrices para implementar políticas públicas a través de programas, proyectos u acciones tendientes a garantizar la equidad entre hombres y mujeres, que toma como base la historia de dominación o exclusión que se haya presentado en razón del sexo, género, orientación sexual o identidad de género.

El enfoque de género se materializa en la actuación del Estado o de las diferentes entidades $u$ organizaciones sociales ya sean públicas o privadas cuando realizan acciones de intervención a partir de la reflexión de cómo están impactando las diferentes políticas a las mujeres o a los hombres y esto incluye a las personas de la comunidad LGBTI.

El enfoque de género se apoya en el enfoque diferencial cuando tiene en cuenta la diversidad de la población según su sexo, edad, etnia, clase social, discapacidad, orientación sexual, identidad de género o territorio en el que habita, y responde al enfoque de derechos humanos en la medida que garantiza a toda la población sin ningún tipo de distinción el acceso a los mínimos vitales consagrados en la Declaración Universal de Derechos Humanos o en la Constitución Política. La Unidad para la Atención y Reparación Integral a las Victimas (s.f.) establece un concepto de enfoque diferencial y de género entendiéndolo como:

una perspectiva integrada de análisis, reconocimiento, respeto y garantía de derechos, enfatiza la diferencia desde la diversidad y reclama la igualdad y la no discriminación. Debe permear la política pública e incidir en las acciones del Estado, pero también incorporarse en los estudios y análisis institucionales y académicos y divulgarse y promocionarse para un cabal reconocimiento y respeto de todos los grupos que integran la nación pluriétnica y multicultural. (p.6)

\section{Políticas públicas}

Las políticas públicas comprenden el conjunto de programas, acciones o directrices en relación al abordaje de una problemática específica con el fin de generar transformaciones sociales de interés colectivo, al respecto Roth (2010) las define como:

Un conjunto conformado por uno o varios objetivos colectivos considerados necesarios o deseables, y por medios y acciones, que son tratados, por lo menos parcialmente, por una institución u organización gubernamental con la finalidad de orientar el comportamiento de actores individuales o colectivos para modificar una

situación percibida como insatisfactoria o problemática. (p.21)

Al hablar de políticas públicas para la paz, se debe procurar vincular a los diferentes grupos sociales en la construcción y definición de las acciones a seguir para resolver el conflicto que se ha presentado, procurando la garantía de derechos para todos, teniendo en cuenta el contexto y las condiciones del territorio, respondiendo a las causas estructurales de la violencia y vincular a las partes en disputa para que se genere mayor compromiso en el cumplimiento de lo pactado.

Una política de construcción de paz y reconciliación que señale la posibilidad de la realización incremental de los derechos, pero sobre todo que genere la capacidad de fortalecer las identidades y capacidades de la gente con fuerza creadora, orientada por la negación absoluta de la violencia como medio de lucha, y búsqueda de la prevalencia de los derechos y una idea de justicia que potencie la multiculturalidad y el respeto por las generaciones futuras, en aras de un mundo mejor (Olmedo, 2011, p.32).

\section{ABORDAJE DEL ENFOQUE DE GÉNERO EN LAS POLÍTICAS PÚBLICAS PARA LA} PAZ EN EL PLANO INTERNACIONAL

A nivel internacional Colombia ha suscrito diversos convenios para garantizar los derechos de las mujeres, mismos que le han instado a incorporar la perspectiva de género en el marco legislativo, en relación a las políticas públicas para la paz expresamente este llamado lo hace Naciones Unidas (1996) al comprometer a los Estados a:

a) Promover la participación de la mujer en condiciones de igualdad y la igualdad de oportunidades para la participación de la mujer en todos los foros y actividades en pro de la paz a todos los niveles... b) Integrar una perspectiva de género en la solución de los conflictos armados o de otra índole... c) Hacer que estos órganos puedan tratar 
debidamente las cuestiones relacionadas con el género impartiendo la formación apropiada a los fiscales, a los magistrados y a otros funcionarios que se ocupan de los casos relativos a violaciones, embarazos forzados en situaciones de conflicto armado, atentados al pudor y otras formas de violencia contra la mujer en los conflictos armados, incluyendo el terrorismo, e integrar una perspectiva de género en su labor. (pp.62-63)

Sumado a lo anterior, Colombia está comprometida con la implementación de la Resolución 1325 de $2000^{4}$ del Consejo de Seguridad de Naciones Unidas - CSNU que insta a los Estados partes a promover acciones locales de construcción de paz desde las organizaciones de mujeres, promover el acceso a la justicia a mujeres víctimas de violencia sexual en el marco del conflicto armado e incorporar la participación de las mujeres en los procesos de paz en el posconflicto, esto implica adoptar perspectiva de género para identificar:

a) Las necesidades especiales de las mujeres y las niñas durante la repatriación y el reasentamiento, asi como para la rehabilitación, la reintegración y la reconstrucción después de los conflictos; b) Medidas para apoyar las iniciativas de paz de las mujeres locales y los procesos autóctonos de solución de conflictos y para hacer participar a las mujeres en todos los mecanismos de aplicación de los acuerdos de paz; c) Medidas que garanticen la protección y el respeto de los derechos humanos de las mujeres y las niñas, particularmente. (Res. 1325, 2000, art. 8 )

Todo esto pone de manifiesto que el enfoque de género ha sido abordado desde la perspectiva de género y además ha sido una preocupación que viene de la mano de la protección internacional a los derechos de las mujeres y de las grandes luchas que ha realizado el movimiento de mujeres en todo el mundo por una sociedad que las incluya y visibilice. Lo anterior evidencia, que la visión internacional en relación con el enfoque de género ha estado pensada desde la lógica femenina, lo cual en sí mismo no está mal, pero sí se constituye en una limitación para su abordaje, interpretación, análisis y

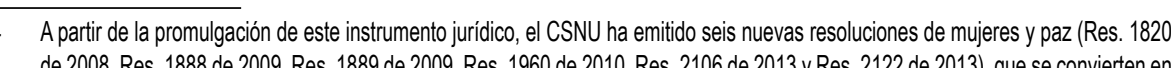
de 2008, Res. 1888 de 2009, Res. 1889 de 2009 , Res. 1960 de 2010 , Res. 2100 de 2013 y Res. 2122 de 2013 , que se convierten en

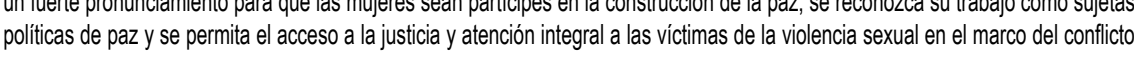
armado. comprensión que sesga el accionar de quien la implementa. Como lo señala Facio:

En el ámbito de las políticas públicas hay una tendencia creciente a crear políticas de género cuando en la práctica se trata de políticas dirigidas a mujeres que dejan intactas las estructuras y relaciones entre los géneros. Una política de género necesariamente tiene que tener como finalidad la superación de la desigual valoración y el desigual poder entre los géneros. De esta manera las políticas públicas de género no necesariamente deberían referirse exclusivamente a las mujeres, en tanto el sistema de género es un sistema relacional que involucra a los varones. Perfectamente podrian haber políticas penitenciarias, por ejemplo, dirigidas a los hombres privados de libertad que podrían ser políticas de género si su objetivo es transformar la forma como los reos ejercen su masculinidad. (s.f., p.19)

\section{ENFOQUE DE GÉNERO EN LAS POLITICAS PÚBLICAS PARA LA PAZ A NIVEL}

\section{NACIONAL}

En agosto del año 2012, el Gobierno Nacional hizo anuncio de una agenda común con las FARC-EP para poner fin al conflicto armado entre las partes y contribuir a la consecución de la paz; dicha voluntad se hizo posible en noviembre de 2016 con la firma del acuerdo final para la terminación del conflicto y la construcción de una paz estable y duradera. Este será el punto de partida para el análisis de la incorporación del enfoque de género en las recientes políticas públicas para la paz en Colombia.

El acuerdo plantea propuestas alrededor de seis puntos: el 1ero, Hacia un nuevo campo colombiano: Reforma Rural Integral; 2do, Promover la Participació política: Apertura democrática para construir la paz; el 3ero, Cese al fuego y de hostilidades bilateral y definitivo y la dejación de las armas; el 4to, Solución al problema de las drogas ilícitas; el 5to, Víctima; y el 6to Implementación, verificación y refrendación.

El acuerdo está planteado desde la lógica de tres enfoques que se encuentran y entrelazan según las condiciones de la población y del territorio, así: enfoque territorial, el conflicto ha impactado de forma diferente los territorios, el 
contexto de las comunidades era diferente antes de ocurridos los hechos de violencia y aún después las consecuencias fueron marcadas por esas particularidades que le hacian único, entonces el enfoque territorial supone tener en cuenta esas características, situaciones, carencias y debilidades que se tenía; requiere reconocer los hechos victimizantes, las víctimas y las afectaciones que generó en el entorno para a partir de allí, entrar a proponer acciones de reparación que involucren a todos desde sus perspectivas y lógicas;

El enfoque diferencial del acuerdo final involucra las afectaciones de los diferentes grupos de poblaciones para desde las lógicas de esas diferencias abordar las políticas, programas y proyectos de atención y reparación que surjan en la implementación del acuerdo; en ese sentido tienen en cuenta a:

... las mujeres, de los grupos sociales vulnerables como son los pueblos indígenas, las niñas, niños y adolescentes, las comunidades afrodescendientes y otros grupos étnicamente diferenciados; de los derechos fundamentales de los campesinos y campesinas y de los derechos esenciales de las personas en condición de discapacidad y de los desplazados por razones del conflicto; de los derechos fundamentales de las personas adultas mayores y de la población LGBTI. (p.3)

Por su parte, el enfoque de género, que trae el acuerdo final crea "condiciones para que mujeres, y personas con identidad sexual diversa puedan acceder en igualdad de condiciones a los beneficios de vivir en un país sin conflicto armado" (Mesa de conversaciones, 2016, p.1), para ello se tuvieron en cuenta los aportes de "organizaciones de mujeres, de la comunidad LGBTI, de las 10 expertas colombianas en violencia sexual, de las expertas internacionales y de las exguerrilleras de varias partes del mundo" (p.1)

a incorporación del enfoque de género en el acuerdo final es el resultado del trabajo de incidencia que realizaron las organizaciones de mujeres y de víctimas por el cumplimiento de lo pactado en la Resolución 1325 de 2000, petición que fue posible con la instalación de la subcomisión de género "que busca hacer recomendaciones a la Mesa que hagan posible un adecuado enfoque de género, particularmente con lo relacionado con las mujeres y la comunidad LGBTI" (Mesa de conversaciones, 2014, p.1), para ello se revisaron y realizaron ajustes en todos los puntos del acuerdo.
INCORPORACIÓN DEL ENFOQUE DE GÉNERO EN LOS PUNTOS

\section{DEL ACUERDO FINAL}

\section{Enfoque de género y reforma rural}

En el punto de Reforma Rural Integral se rige entre otros por el principio de igualdad y enfoque de género para el bienestar y buen vivir de las personas y hace énfasis en acciones afirmativas en favor de las mujeres en tanto se propone la implementación de los planes y programas, así:

- Reconocimiento de las condiciones socioeconómicas y culturales de las mujeres.

- Acceso en condiciones de igualdad de hombres y mujeres a los proyectos productivos, créditos, propiedad de la tierra, procesos de formación, servicios técnicos e infraestructura.

- Acceso a los servicios de salud sexual y reproductiva acorde a los ciclos de vida, con especial énfasis en mujeres gestantes en el Plan Nacional de Salud Rural.

\section{Enfoque de género y participación política}

En relación al punto de Participación política en el marco del Sistema Integral de Seguridad para el Ejercicio de la Política plantea una mirada a la seguridad desde el enfoque territorial, diferencial y de género que permita identificar elementos para la prevención, protección y seguridad individual y colectiva, sobre todo de líderes/as defensores de derechos humanos de organizaciones o movimientos sociales. En ese sentido plantea:

- Fortalecimiento del programa de protección individual y colectivo teniendo en cuenta los riesgos de las personas por su condición diferencial o de género.

- Establecer un diagnóstico con enfoque de género que permita la identificación de los obstáculos para elegir y ser elegidos.

La promoción de la no estigmatización y discriminación de los grupos poblacionales históricamente excluidos para la reconciliación y una mejor convivencia.

- La realización de campañas de sensibilización y comunicación para la democracia y garantía de derechos que visibilice las múltiples formas 
de discriminación de los grupos poblacionales haciendo énfasis en las minorías étnicas, religiosas o de género.

- El rechazo a las discriminaciones que han sufrido las mujeres, el reconocimiento del género femenino como sujetos políticos que han contribuido a la democracia y la paz.

- La adopción de medidas para el fortalecimiento de los liderazgos y la participación de la mujer.

\section{Enfoque de género y cese al fuego y de hostilidades bilateral y definitivo y}

\section{la dejación de las armas}

Por su parte, en el del Cese al fuego y de hostilidades bilateral y definitivo y la dejación de las armas establece medidas relacionadas con el punto 2 sobre Participación política y en razón a ellos señala:

- La adopción del enfoque diferencial y de género en la implementación de medidas relacionadas con la seguridad y el ejercicio de la participación política.

- Como principio para las garantías de seguridad, fortalecimiento de la administración de justicia que responda a las demandas y casos de violencias basadas en género, de discriminación o estereotipos sobre la población LGBTI para garantizar el acceso a la justica y la sanción debida.

- La realización de un Pacto Político Nacional, que incluya entre otros actores a organizaciones sociales de mujeres y de población LGBTI.

- El enfoque de género en la Comisión Nacional de Garantías de Seguridad para el diseño, implementación y seguimiento de políticas públicas que permita identificar actividades criminales y financiación de las mismas en contra de las mujeres y población LGBTI.

Que la Unidad Especial de Investigación cuente con funcionarios especializados en temas de justicia y violencia de género para identificar situaciones que ponen en peligro a las comunidades.

- Esquemas de seguridad y protección con enfoque de género para el nuevo movimiento o partido político de las FARC-EP.

- Atención psicosocial con enfoque de género a destinatarios de programas de protección.

- La incorporación del enfoque territorial, diferencial y de género en el Sistema de Alertas Tempranas.

\section{Enfoque de género y problema de las drogas ilícitas}

En cuanto al punto de Solución al problema de las drogas ilícitas el acuerdo final señala que la pobreza y el abandono han incidido de manera negativa en el desarrollo de los territorios, lo que ha llevado a pobladores a cultivar, producir y comercializar drogas ilícitas, y esto a su vez ha sido un detonante de la violencia basada en el género, razón por la cual las políticas públicas de atención a la problemática deben incorporar un enfoque de derechos humanos, diferenciado y de género, ( $p .99)$, así:

Debe reconocer y tener en cuenta las necesidades, características y particularidades económicas, culturales y sociales de los territorios y las comunidades rurales, en especial de las comunidades indigenas y afrodescendientes, y de las mujeres en estas comunidades y territorios, y garantizar la sostenibilidad socioambiental. (p.103)

El acuerdo contempla la creación de un Programa Nacional Integral de Sustitución - PNIS, que involucre la participación de las mujeres en el diseño, implementación y evaluación de los planes relacionados con la sustitución y desarrollo alternativo, igualmente contempla como principio:

Enfoque diferencial y de género: en el marco del respeto a los derechos humanos, para que las acciones que se implementen en materia de consumo respondan a las realidades de los consumidores y las consumidoras y sean efectivas y sostenibles, es necesario identificar factores de vulnerabilidad asociados a edad, sexo, condición de discapacidad, condición socioeconómica y ubicación geográfica o pertenencia a la población LGBTI, entre otros... Este enfoque deberá tener en cuenta la relación entre el consumo de las drogas ilícitas y la violencia contra la mujer, especialmente con la violencia intrafamiliar y la violencia sexual. Se preverán medidas para mujeres, niñas, jóvenes y adolescentes... (p.117)

Asimismo, se establece el diseño y funcionamiento de un Sistema Nacional de Atención a las Personas Consumidoras de Drogas Ilícitas, que maneje el enfoque de género: en el tratamiento y rehabilitación de consumidores/as y permita hacer el vínculo con la violencia intrafamiliar, violencia sexual y el VIH SIDA; en las investigaciones que realice, y en las acciones de sensibilización. 


\section{Enfoque de género y víctimas}

En referencia al punto de Víctimas, el acuerdo final hace relación al impacto desproporcionado del conflicto en los diferentes grupos poblacionales, dentro de ellos, las mujeres y población LGBTI, en consecuencia, establece la conformación del Sistema Integral de Verdad, Justicia, Reparación y No Repetición - en adelante SIVJRNR, que tenga en cuenta el enfoque diferencial y de género para dar tratamiento diferenciado según los hechos victimizantes.

El Sistema Integral contempla la creación de la Comisión para el Esclarecimiento de la verdad, la convivencia y la no repetición, que debe responder entre otras al conocimiento de los hechos de violencia basada en género realizados en el marco del conflicto armado y evidenciar las afectaciones específicas en mujeres y población LGBTI, para eso "tendrá en cuenta las distintas experiencias, impacto diferencial y condiciones particulares de las personas, poblaciones o sectores en condiciones de discriminación, vulnerabilidad..." (p.133), todo ello para el esclarecimiento de los hechos y la promoción del reconocimiento de la verdad. Dentro de las funciones de la Comisión está el:

Asegurar la transversalidad del enfoque de género en todo el ámbito de trabajo de la Comisión, con la creación de un grupo de trabajo de género que contribuya con tareas específicas de carácter técnico, de investigación, preparación de audiencias, entre otras. Este grupo de trabajo no será el único en tratar el tema, pero sí debe responsabilizarse de la revisión de metodologías para que todos los instrumentos de la Comisión tengan este enfoque, y de la coordinación con organizaciones de mujeres y LGBTI... (p.137)

En el punto de Víctimas, se contempla además la conformación de un Comité de seguimiento y monitoreo a la implementación de las recomendaciones de la Comisión, que debe emitir informes periódicamente que incluyan el enfoque territorial, diferencial y de género.

Seguidamente, en el marco de la Jurisdicción Especial para la Paz - JEP, Se proponen unos principios orientadores dentro de los cuales se encuentra el cumplimiento de lo pactado con Naciones Unidas en relación al enfoque de género y la participación de las mujeres en procesos de onstrucción de paz, de tal manera que haga énfasis en las necesidades y consecuencias del conflicto en la vida de las niñas, mujeres, población LGBTI y otras víctimas.
Los Planes nacionales de reparación colectiva, que incluyen el punto de Victimas, reconoce la importancia de la participación de las organizaciones sociales de mujeres para el desarrollo, la convivencia y la reconciliación; en cuanto a los procesos colectivos de retorno y restitución de tierras señala la necesidad de incorporar el enfoque de género.

Cabe agregar que el acuerdo final señala que para garantizar los derechos humanos a todas las personas se debe incluir el enfoque de género en la construcción de la paz y ello requiere de acciones afirmativas para superar la discriminación de grupos históricamente excluidos y oprimidos.

\section{Enfoque de género e implementación, verificación y refrendación}

Finalmente, en el punto de Implementación, verificación y refrendación, se expone que "El enfoque de género deberá ser entendido y aplicado de manera transversal en la implementación de la totalidad del Acuerdo" (p.194) para ello se garantizan como principios la igualdad y no discriminación, en lo que respecta a los grupos poblacionales que incluye a personas LGBTI el enfoque de género íntimamente relacionado con las problemáticas que atañen a las mujeres.

\section{El enfoque de género en la Ley 1448 de 2011}

Si bien la Ley 1448 de 2011 del Congreso de la República surge previo a las negociaciones públicas entre el Gobierno colombiano y las FARC-EP, empero e acuerdo final en el punto 6 sobre Implementación, verificación y refrendación enfatiza la necesidad de hacer modificaciones a esta norma "con base en lo acordado en el punto 5.1.3.7 del acuerdo de "Víctimas", teniendo en cuenta el principio de universalidad y conforme a los estándares internacionales" (p.203).

En ese sentido la Ley 1448 de 2011, conocida como Ley de Víctimas y restitución de tierras, referencia el enfoque de género a partir de la igualdad, con el respeto a la libertad de orientación sexual en su artículo 6; como criterio de implementación en el artículo 32; como obligación para la asesoría judicial de victimas (art. 35); en la atención preferencial para las mujeres en los trámites administrativos o judiciales (art. 114); y en el acompañamiento psicosocial para la rehabilitación (art. 136). 
Específicamente en el artículo 13, como principio de intervención en todas las medidas señaladas en la ley, se hace alusión al enfoque diferencial, señalando aspectos relacionados con el enfoque de género, asi "hay poblaciones con características particulares en razón de su edad, género, orientación sexual y situación de discapacidad".

\section{El enfoque de género en la Sentencia C579 de 2013}

La Sentencia C-579 de 2013 de la Corte Constitucional de Colombia, sobre el marco jurídico para la paz, supone el enfoque de género como un compromiso internacional y del Estado Social de Derecho que obliga a respetar el goce de los derechos humanos a todas las personas sin discriminaciones que "condicionen el acceso o el ejercicio de los derechos, basadas en criterios tales como el género, la nacionalidad y la casta".

\section{El enfoque de género en la Ley 1719 de 2014}

La Ley 1719 de 2014 del Congreso de la República adopta "medidas para garantizar el acceso a la justicia de las víctimas de violencia sexual, en especial la violencia sexual con ocasión del conflicto armado" (Art. 1), surge durante las negociaciones Gobierno colombiano y las FARC-EP y evidencia un compromiso del Estado con las víctimas de violencia sexual.

Esta ley materializa el enfoque de género a través de:

- La creación de Comités Técnico-Jurídicos para la Investigación de la Violencia Sexual al interior de la Fiscalía General de la Nación, el cual realizará "...análisis, monitoreo y definición de técnicas y estrategias de investigación con perspectiva de género y diferencial..." (Art. 21).

- La exigencia de que quienes hacen parte de los comités tengan experiencia y estén formados en temas de derechos humanos, enfoque diferencial y de género (Art. 21)

- Las medidas de reparación que deben responder a "...criterios diferenciales de edad, grupo étnico, orientación sexual, identidad o expresión de género..." (Art. 25) entre otros.

- El fortalecimiento de la "política en derechos sexuales y reproductivos, salud sexual y reproductiva, equidad y violencia basada en género" (Art. 30).
- La "incorporación al Sistema de Registro Unificado de Casos de Violencia contra la Mujer" (Art. 31), que evidencie:

1. El lugar y la fecha de ocurrencia de los hechos. 2. Caracterización de las víctimas, especificando el sexo, edad, grupo étnico, orientación sexual, identidad o expresión de género, condición de discapacidad, condición de desplazamiento forzado o de víctima de conflicto armado, pertenencia a una organización social, actividad de liderazgo, entre otros. 3. Caracterización del presunto victimario especificando el sexo, la edad, pertenencia a un grupo armado y su identificación, relación con la víctima, entre otros criterios diferenciales. 4. Medidas de prevención, atención y protección adoptadas. 5. Casos que son conocidos por las autoridades judiciales, si se ha presentado denuncia, calificación jurídica provisional o definitiva, etapa del proceso penal y existencia de fallos sobre responsabilidad penal. (Art.31)

\section{El enfoque de género en el Decreto 1995 de 2016}

Atendiendo a las directrices del acuerdo final, el Gobierno Nacional promulga el Decreto 1995 de 2016, que auspicia la creación de la Comisión de Seguimiento, Impulso y Verificación a la Implementación - CSIVI, la cual debe tener una "Instancia Especial para el seguimiento al enfoque de género y garantía de derechos de las mujeres en la implementación del acuerdo final" (Art. 6).

En razón a ello, en comunicado conjunto \# 18 de la Presidencia de la República se emiten los criterios generales para Instancia Especial, que estará conformada por siete organizaciones de mujeres de nivel nacional y territorial, dentro de las que se encuentran además una organización de víctimas y una de población LGBTI. La instancia se regirá por los principios del:

Enfoque de derechos; Respeto a la igualdad y no discriminación; Enfoque de género; Respeto a libertad de cultos; Integración territorial e inclusión social; Fortalecimiento y articulación institucional; Profundización de la democracia y "construir sobre lo construido"; Eficacia, eficiencia e idoneidad; Priorización; Transparencia, contro social y lucha contra la corrupción; y Principios democráticos. Adicionalmente, se orientará por el enfoque de interseccionalidad. (p.2) 
Dentro de las funciones de la instancia, estará el realizar las recomendaciones necesarias y hacer seguimiento a través de informes periódicos para el cumplimiento del enfoque de género en lo relacionado con las políticas, planes o programas que estén de la mano de la implementación del acuerdo; mantener comunicación permanente para el establecimiento de propuestas y adecuado seguimiento "... con las mujeres y las organizaciones, plataformas, colectivos, grupos y redes, cumbres o movimientos a nivel nacional y territorial..." (p.3) y "con la CSIVI, el Consejo Nacional de Reincorporación (CNR), los componentes de verificación y acompañamiento internacional..., el Instituto Kroc y demás instancias establecidas por el acuerdo final..." (p.3).

\section{El enfoque de género el Acto Legislativo № 1 de 2017}

El Acto Legislativo № 1 de 2017 del Congreso de la República reglamenta las disposiciones generales del acuerdo final sobre víctimas, dentro del cual se incluye el Sistema Integral de Verdad, Justicia, Reparación y No Repetición - SIVJRNR y la Jurisdicción Especial para la Paz - JEP, y específicamente en el parágrafo 1 del artículo transitorio 1 y 12 sobre el SIVJRNR y JEP respectivamente, recalca la obligación de implementar en todo el proceso el enfoque de género, atendiendo las diferencias entre los sexos y las afectaciones especificas de las mujeres.

\section{Enfoque de género en acción}

Como se ha podido evidenciar en los diferentes instrumentos jurídicos señalados hasta el momento el enfoque de género que se plantea en las políticas públicas para la paz, se basa en el reconocimiento de las diferencias de sexo, orientación sexual y hace especial énfasis en las problemáticas contra las mujeres relacionadas con las violencias y dificultades para acceder a la participación política. En cuanto a identidades de género solo la Ley 1719 de 2014, sobre violencias sexuales plantea esta categoría de análisis e intervención.

En la práctica, la Alta Consejería Presidencial para la Equidad de la Mujer (2012), establece el género como "categoría de análisis social que permite comprender, en parte, las relaciones entre hombres y mujeres, en especial, cómo operan estas en múltiples campos incluidos el simbólico, a través de representaciones, en los conceptos normativos, en las instituciones, en las organizaciones sociales y en las identidades subjetivas (p.49).
Por tanto, consideran importante que las PP de género sean transversalizadas en el accionar político, legislativo y práctico de las entidades públicas, en tanto pueda ser utilizado como "herramienta para hacer de los intereses y necesidades de hombres y mujeres una dimensión integrada en el diseño implementación, monitoreo, y evaluación de planes y programas en todos los ámbitos políticos, sociales y económicos" (p.118).

Todo lo anterior lleva a establecer una serie de recomendaciones para la implementación del enfoque de género en las políticas públicas para la paz, aclarando que, al mencionar la palabra hombres o mujeres, se trata de hacer referencia a toda la población teniendo en cuenta su diversidad sexual, cultural, étnica, política, religiosa, de clase, de edad, identidad, orientación o territorio, es decir en plural, no teniendo un único patrón o modelo. Es así que el enfoque se puede implementar cuando:

- Se reconocen las discriminaciones que sufren algunos grupos poblacionales basadas en la historia de dominación patriarcal que legitima la diferencia de sexo y género y a partir de allí les excluye, reprime, violenta o invisibiliza.

- Se apoya en el enfoque diferencial a fin de realizar un análisis integral de las situaciones problemas que se puedan presentar y contribuye a garantizar en esa medida el enfoque de derechos para todas las personas.

- Se analizan los hechos victimizantes del conflicto armado a la luz de la incidencia que pudo tener el ser hombres o mujeres, pero también al hacer parte de la comunidad LGBTI.

Se identifica el impacto desproporcionado del conflicto armado para hombres o mujeres en su diversidad.

- Se analiza el papel de las mujeres y hombres en los distintos espacios de la esfera pública o privada ya sea en lo familiar, social, cultural, educativo, económico, laboral, científico, político u otro.

Se toman directrices en relación con los diferentes espacios mencionados en el ítem anterior, de evidenciarse en ellos algún tipo de discriminación basada en el sexo o el género. 
- Se promueven acciones tendientes a garantizar los derechos, sobre la base de las afectaciones que le ocurrieron según su sexo 0 género.

- Se utiliza un lenguaje incluyente, libre de discriminaciones, estereotipos, señalamientos, prejuicios, burlas u ofensas contra alguna persona, y por ende se rechaza todo tipo de insinuaciones degradantes del ser humano.

- Se tolera la libre determinación de ejercer la sexualidad, así como el proceso de reconocimiento de la identidad de género, esto incluye respetar su orientación sexual, las prácticas que realiza y el cómo desea ser llamada.

Con el fin de complementar un poco más esta lista de recomendaciones, vale la pena señalar que la Unidad de Atención y Reparación Integral a las Victimas (2016), manifiesta que han recibido "quejas contra servidores públicos por haber aconsejado a la víctima cambiar, moderar u ocultar su orientación o dentidad de género, o sugerir que el hecho victimizante esté justificado por la orientación sexual o expresión de la identidad género" (p.7), lo anterior evidencia discriminación y debe ser una conducta reprochable y sancionable en la medida que legitima la conducta violenta, justifica la agresión y culpabiliza a la víctima. Igualmente exhortan a hacer:

... siempre las preguntas sobre identidad de género y orientación sexual sin asumir la respuesta por la apariencia física, comportamientos 0 ademanes de la persona. Tenga en cuenta que no es ofensivo indagar por la orientación sexual, pero sí lo es pasar por alto las necesidades diferenciales. (p.8)

La misma entidad en la Guía operativa para la implementación de acciones de Enfoque Diferencial y de Género en los procesos de asistencia y reparación a las víctimas (2016), en los casos en los que la víctima sea una mujer, invita a que se:

... Explique a las mujeres que: Solo se hablará de lo que la mujer desea. Se indagará sobre las preguntas que están contempladas dentro del proceso y no se ahondará en detalles de la intimidad y privacidad. Está en plena libertad de expresar sus sentimientos. Evitar ahondar sobre la ocurrencia del hecho en los casos de violencia sexual. Evitar emitir juicios de valor $u$ opiniones de 10 sucedido y de lo que pudo o no haber hecho la mujer víctima de violencia sexual, no hacer observaciones que justifiquen cualquier acto de violencia contra las mujeres. (p.19)

Por su parte, en casos cometidos contra personas con orientaciones sexuales e identidades de género, diversas, se procure:

Mantener una actitud libre de prejuicio. Esto implica entender que las orientaciones sexuales e identidades de género no hegemónicas no son enfermedades, contravenciones a la legislación ni acciones indebidas ni contagiosas. Recordar que en virtud de la normatividad antidiscriminación (Ley 1482 2011) está terminantemente prohibido cualquier acto discriminatorio... Las creencias religiosas no deben interferir en la atención, teniendo en cuenta el carácter laico del Estado... (p.20).

\section{CONCLUSIONES}

No se puede hablar de paz mientras existan estructuras patriarcales de opresión, violencia y discriminación contra las mujeres, mientras no se acepte al otro en su diferencia, en su libre ejercicio de la sexualidad y libre identidad de género, sin importar estar de acuerdo o no, simplemente respetando que tiene derecho a pensar, sentir y vivir de una forma diferente de la forma como escogió ser y estar en el mundo.

El enfoque de género además de verse desde la interseccionalidad, debe analizarse y aplicarse para que los hombres también hagan parte; es necesario reevaluar el enfoque de género en la concepción referente al hombre, dado que problemáticas como las pandillas, las drogas, los suicidios entre otros, tienen una fuerte carga patriarcal, que debe ser analizada a la luz de la distribución de roles en la sociedad y que, de no ser analizadas podrían terminar siendo discriminatorias contra el género masculino.

Si bien en relación al acuerdo de paz se han establecido otras normas de políticas públicas para la paz - Ley 1732 de 2014, no aborda el enfoque de género. Así mismo se requiere que se elaboren protocolos de implementación que acompañen las normas ya promulgadas $u$ otras que surjan en el marco del cumplimiento de lo pactado en el acuerdo final. 


\section{REFERENCIAS BIBLIOGRÁFICAS}

Alta Consejería Presidencial para la Equidad de la Mujer (2012). Lineamientos de la Política Pública Nacional de Equidad de Genero para las Mujeres. Recuperado de ww.equidadmujer.gov.co/.../Lineamientos-politica-publica-equidad-de-genero. df

Caribe Afirmativo (2010). Manual para el reconocimiento de derechos de la diversidad sexual e identidad de género. Cartagena: Editorial Caribe Afirmativo.

comisión de Seguimiento, Impulso y Verificación a la Implementación del Acuerdo final (2017). Comunicado conjunto No. 18. Recuperado de: es.presidencia.gov.co/ Documents/170411_ComunicadoConjunto18.pdf

Congreso de la República de Colombia (10 de junio de 2011). Ley de Víctimas y Restitución de Tierras [Ley 1448 de 2011]. DO: 48.096.

congreso de la República de Colombia (18 de junio de 2014). Ley de Atención a Víctimas de Violencia Sexual [Ley 1719 de 2014]. DO: 49.186.

Congreso de la República de Colombia ( 4 de abril de 2017). Acto Legislativo sobre lusticia Especial para la Paz. [Acto Legislativo 1 de 2017]. Recuperado de: http:// es.presidencia.sov.co/normativa/actos-legislativos

Consejo de Seguridad de Naciones Unidas (31 de octubre de 2000). Resolución 1325 de 2000. Recuperado de: http://www.un.org/es/peacekeeping/issues/women/wps. shtml

Corte Constitucional de Colombia. Sentencia C-579 de 2013. Recuperado de: http:// www.corteconstitucional govco/relatoria/2013/C-579-13.htm

Facio, A. (s.f.). Lectura de Apoyo 1: Feminismo, género y patriarcado. Costa Rica.

Mesa de Conversaciones para la Terminación del Conflicto y la Construcción de una Paz Estable y Duradera en Colombia (2014). Comunicado conjunto No. 41. La Habana Cuba. Recuperado de: htlps.//Ww.mesadeconversaciones.com.co/comunicados/ comunicado-conjunto-la-habana-11-de-septiembre-de-2014

Mesa de Conversaciones para la Terminación del Conflicto y la Construcción de una Paz Estable y Duradera en Colombia (2016). Comunicado conjunto No. 82. La Habana Cuba. Recuperado de. htps.//www.mesadeconversaciones.com.co/comunicados/ comunicado-conjunto-82-la-habana-cuba-24-de-julio-de-2016

Mesa de Conversaciones para la Terminación del Conflicto y la Construcción de una Paz Estable y Duradera en Colombia (2016). Acuerdo Final para la Terminación del Conflicto y la Construcción de una Paz Estable y Duradera. Bogotá. Recuperado de: https://ww.mesadeconversaciones.com.co/comunicados/acuerdo-fnal-para-la-terminaci\%c3\%B3n-det-comlicto-y-la-construl table-y-1

Naciones Unidas (1996). Informe de la Cuarta Conferencia Mundial sobre la Mujer. Beijing. Recuperado de: www.un.org/womenwatch/daw/beijing/pdf/Beijing\%20

Olmedo, L. (2011). Reflexiones para la construcción de políticas públicas para la paz considerando el enfoque de acción sin daño y construcción de paz. Universidad Nacional de Colombia, Bogotá.

Presidencia de la República de Colombia (7 de diciembre de 2016). Comisión de Seguimiento, Impulso y Verificación a la implementación del Acuerdo Final. [Decreto 1995 de 2016]. Recuperado de. http://es.presidencia.gov.co/normativa/decretos2016/decretos-diciembre-2016

Roth, A. (2010). Enfoques para el análisis de políticas públicas. Universidad Nacional de Colombia, Bogotá.
Unidad para la Atención y Reparación Integral a las Víctimas (s.f.). Enfoque étnico pueblos y comunidades indigenas, comunidades negras, afrocolombianas, raizales, palenqueras y pueblo Rrom. Recuperado de: https://www.unidadvictimas. gov.co/sites/default/files/.../enfoqueetnico.PDF

Unidad para la Atención y Reparación Integral a las Víctima (2016). Orientación sexual e identidad de género Recuperado de. https://www.unidadvictimas.gov.co/sites/ default/files/.../sexualidad_0.pdf 